\title{
SABERES EDUCATIVOS MAPUCHES: RACIONALIDAD APOYADA EN LA MEMORIA SOCIAL DE LOS KIMCHES
}

\author{
MAPUCHE EDUCATIONAL KNOWLEDGE: RATIONALITY \\ BASED ON THE SOCIAL MEMORY OF KIMCHES
}

\section{DANIEL QUILAQUEO R.2}

\section{RESUMEN}

Los saberes educativos que se utilizan para la formación de niños, niñas y jóvenes mapuches se sustentan en la memoria social de los kimches (sabios). Estos saberes se desprenden de una racionalidad que ha sido exitosa en la formación de personas y en la enseñanza de los contenidos sociales y culturales. Es decir, son contenidos que cobran sentido y que se abordan desde la coherencia que los kimches le otorgan a sus discursos educativos, a través de métodos específicos y de valores propios y universales. En consecuencia, desde un punto de vista epistemológico, jurídico y ético, el currículum escolar puede incorporar los conocimientos educativos vernáculos en las escuelas situadas en contextos mapuches. Finalmente, entre los resultados que se presentan en el estudio se revelan las categorías conceptuales de Kimeltuwün (acción educativa), Kimün (saberes) y Prácticas Socioculturales.

Palabras clave: Saberes educativos mapuches, formación de persona, racionalidad $k i$ mche.

\section{ABSTRACT}

The educational knowledge used for raising Mapuche children and adolescents is based on the social memory of the kimches (sages). This knowledge derives from a rationality which has been successfully applied in raising children and in teaching them social and

\footnotetext{
${ }^{1}$ Este artículo presenta resultados del proyecto Fondecyt Regular No 1085293 "Racionalidad del método educativo mapuche desde la memoria social de kimches: fundamentos para una educación intercultural".

${ }^{2}$ Doctor en Sociología, director del Núcleo Milenio "Centro de Investigación en Educación en Contexto Indígena e Intercultural” (CIECII), Facultad de Educación, Universidad Católica de Temuco.Temuco, Chile.E-mail: dquilaq@uct.cl
} 
cultural contents. In other words, these contents make sense and are addressed from the coherence which the kimches impart to their educational discourse, through specific methods and their own and universal values. Consequently, from an epistemological, legal and ethical standpoint, the school curriculum can perfectly well incorporate vernacular educational knowledge in schools situated in Mapuche contexts. Finally, among the results presented in the study, the conceptual categories of Kimeltuwün (educational action), Kimün (knowledge) and Sociocultural Practices are revealed.

Keywords: Mapuche educational knowledge, bringing up young people, kimche rationality.

Recibido: 24.09.10. Aceptado: 28.05.11.

\section{INTRODUCCIÓN}

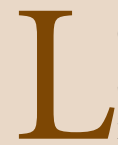

OS SABERES EDUCATIVOS mapuches, que son vitales para el desarrollo cultural y social de las familias y comunidades con una identidad basada en sus propios conocimientos, subsisten entre los kimches de las distintas comunidades territoriales ${ }^{3}$. Estos saberes fueron conocidos por los misioneros católicos durante la colonización española (Molina, 1778; Housse, 1939; Latcham, 1924; Noggler, 1972; Bradford, 1976; Bengoa, 1987). Posteriormente, con la creación de las Escuelas Misionales (católicas y protestantes) y la escuela pública a fines del siglo XIX, los saberes educativos mapuches no fueron considerados en el currículum escolar. Por el contrario, y al igual que lo ocurrido con los pueblos indígenas de América Latina, en la escuela se impuso el modelo de castellanización como el principal medio de integración de los niños y niñas indígenas ${ }^{4}$, lo que se ha justificado con la idea de modernización (Yáñez, 1988).

Desde 1993, la Ley Indígena No 19.253, en Chile, y el Convenio 169 de la Organización Internacional del Trabajo (OIT), en el plano internacional, plantean la incorporación de los saberes vernáculos en las escuelas que

\footnotetext{
${ }^{3}$ Comunidad territorial se refiere al concepto de colectividad histórica (Schnapper, 1998). Este concepto es más pertinente para designar lo que se denomina habitualmente como grupo étnico, lo que permite explicar la ocupación geográfica del territorio y designar las comunidades, por ejemplo, las comunidades situadas en la zona andina reciben la denominación Pewenche; las situadas al lado del Océano Pacífico, Lafkenche; las situadas en la parte norte de la Araucanía, Picunche y las que se sitúan al sur, Williche, de acuerdo con los puntos cardinales.

${ }^{4}$ En Chile se implantó una educación monolingüe y monocultural que favoreció a los hijos de colonos en desmedro de la población indígena. Desde entonces, los padres de las familias indígenas han solicitado al Estado y a la Iglesia Católica la incorporación de sus saberes educativos, conjuntamente con los saberes occidentales que se imparten en la escuela (Noggler, 1972; Quilaqueo, 1994 y 2006).
} 
cuentan con población indígena. Éstos son instrumentos jurídicos que proponen una educación intercultural ${ }^{5}$ como medio para reconocer la cultura de los alumnos de origen indígena (CONADI, 1993; OIT, 1989; UNESCO, 2005). En consecuencia, es legal la incorporación del conocimiento educativo mapuche en las escuelas instaladas en las comunidades y en los nuevos contextos de vida ${ }^{6}$ mapuche.

La incorporación de los saberes educativos al currículum escolar requiere de una base epistemológica que dé cuenta de la sabiduría que proviene de las propias personas que portan el conocimiento educativo y cultural mapuche, lo que implica considerar la racionalidad que le ha permitido al pueblo mapuche subsistir como personas, familias y comunidad con una identidad cultural propia (Quilaqueo, Quintriqueo, Catriquir y Llanquinao, 2004). Las personas que se han formado a partir de saberes y conocimientos culturales lo han hecho mediante mitos, leyendas, cuentos y la historia de su ascendencia paterna y materna, es decir, mediante hechos y contenidos de la memoria familiar y de las comunidades (Quilaqueo, 2007). Entre las personas que cultivan estos saberes se destaca el kimche, porque es quien conoce tanto los saberes educativos como los aspectos sociales y culturales de las familias y de las distintas comunidades territoriales, a partir de lo que se denomina kuifike $z u g u^{7}$. Por lo tanto, es necesario conformar un cuerpo de proposiciones para formular una teoría que contenga los conocimientos y saberes educativos propios de las comunidades mapuches de la Región de la Araucanía.

\section{MARCO TEÓRICO}

Este trabajo se sustenta en estudios socioeducativos (Colom, 1992), lo que permite abordar la educación impartida por los kimches a partir de un enfoque tanto mapuche como no mapuche. Desde esta perspectiva, primero, se

\footnotetext{
${ }^{5}$ En investigaciones sobre educación intercultural en contexto mapuche, se muestra que las familias de las comunidades reclaman una mejor calidad de educación y la incorporación de conocimientos socioculturales mapuches a la vida escolar (Loncón, 1997; MINEDUC, 2003; Quilaqueo, Quintriqueo y Cárdenas, 2005; Quilaqueo, 2007).

${ }^{6}$ Se denomina nuevo contexto de vida mapuche al asentamiento en el medio urbano como efecto de la emigración y, en las dos últimas décadas, en el medio rural, en nuevas localidades fuera de la comunidad consanguínea, como resultado del programa de restitución de tierras por parte del Estado.

${ }^{7}$ La expresión kuifike zugu se traduce como los temas principales del conocimiento ancestral y se puede asociar con el concepto de memoria social.
} 
analiza la educación mapuche considerando los conceptos de kimeltuwün y kimche y, segundo, se consideran enfoques sociológicos y filosóficos sobre el concepto de racionalidad, donde se abordan aspectos tanto epistemológicos como éticos.

El concepto de kimeltuwün, en la racionalidad mapuche, representa la acción educativa que implica un proceso de aprendizaje-enseñanza entre dos o más personas. Este proceso tiene como objetivo comprender y aprender un contenido que ayude a racionalizar las creencias con respecto a la naturaleza, al medio social y a aspectos espirituales (Quilaqueo et al., 2004). El kimeltuwün es primordial para la formación de los niños, niñas y jóvenes y se sustenta en los siguientes principios: 1) el kümeyawal ta che, que es la actitud y relación de respeto que idealmente se debe establecer entre los miembros de la familia y las comunidades mapuches; 2) el yamüwal ta che, que está representado por una relación de estima y de reconocimiento entre personas de un mismo grupo parental y de la comunidad mapuche en general; 3) el küme rakizuam ta nieal, que es el principio que considera el conocimiento de la formación que permite concebir a la persona, tanto al interior de la familia como en la comunidad; 4) el küme che geal, que es el enfoque evaluativo cuyo fin es determinar si la persona actúa solidariamente dentro del grupo parental; 5) y el kim che geal, que es el principio que guía la formación de la persona sobre la base de un conocimiento (Quintriqueo, 2005).

Por su parte, el concepto kimche se refiere a mujeres y hombres que poseen los conocimientos educativos para la formación de personas y de oficios. Este concepto ha sido definido como persona sabia, docta y erudita (Augusta, 1903; Erize, 1960) y, desde el punto de vista socioeducativo, en nuestras investigaciones, como sabio portador del conocimiento educativo. En efecto, el kimche, hoy día, puede definirse como la persona que posee conocimientos mapuches y no mapuches, cuyo objetivo es articular un discurso formativo ${ }^{8}$ para la educación de los niños, niñas y jóvenes (Quilaqueo et al., 2004; Quilaqueo, 2006). En la educación mapuche se reconocen varios conceptos que se relacionan con la enseñanza y el aprendizaje; sin embargo, este trabajo considera principalmente al concepto de kimün,

\footnotetext{
${ }^{8}$ Para identificar los saberes educativos, el concepto de discurso que se ha utilizado se entiende como un complejo de tres elementos interrelacionados e interdependientes entre sí: el texto y sus elementos constitutivos, la práctica discursiva que los hablantes realizan a través de los textos y la práctica social, donde tanto práctica discursiva como texto son modelados ideológicamente (Fairclough 1995; Van Dijk 1997 y Wodak 2003).
} 
que se define, en general, como saber ${ }^{9}$ (Augusta, 1903; Erize, 1960; Catrileo, 1995).

Desde un enfoque sociológico, considerando las proposiciones de Bajoit (2003), se puede señalar que no es posible comprender la vida social actual de los pueblos indígenas si no se pone al sujeto individual como elemento central, donde la vida social implica obligaciones de las comunidades sobre los individuos que forman parte de ella. Esta perspectiva intenta explicar las conductas mapuches colectivas, el orden y el cambio en las comunidades a partir del conocimiento de los kimches. Los sujetos mapuches, que a la vez son sujeto y objeto de sus relaciones sociales, buscan construir y manifestar su identidad personal mediante intercambios que le permiten comprender la vida social. Una de las proposiciones de Bajoit plantea que "la vida social implica apremios del colectivo sobre los individuos que forman parte de éste" (2003: 31). Esto indica que es urgente considerar los saberes educativos mapuches como saberes vitales para el desarrollo del pueblo mapuche como tal ${ }^{10}$, debido a que no se han resuelto los problemas derivados de la escolarización descontextualizada que ha imperado en las escuelas de las comunidades, lo que pone en peligro de desaparición el saber educativo mapuche.

Por su parte, el concepto de racionalidad, en el campo de las ciencias sociales, ha estado vinculado con el estudio de las sociedades, de sus culturas y de su proyecto de vida. En este artículo, el concepto de racionalidad se considera como el medio por el cual la persona busca garantizar y adecuar sus creencias y acciones al contexto, con el objeto de que sea una base segura que le permita tener razones suficientes y válidas para justificar dichas creencias (Quilaqueo, 2010). Esto quiere decir que se espera que exista una aceptación general, común entre las personas de las comunida-

\footnotetext{
${ }^{9}$ Kimün se traduce, además, como conocer, aprender, sentir y adivinar. La palabra kimün proviene de la raíz kim, que en su carácter de adjetivo significa sabido y entendido. Augusta, en su gramática, señala que kim “... es la radical del verbo kimn” (1903: 138), que se traduce como saber, aprender y sentir. Así, la ante sílaba kim porta tres campos de significados. Uno de los significados se vincula con el conocimiento y el saber que, como sustantivo, significa sabiduría; otro de los significados se vincula con lo educativo, que incluye el binomio enseñar y aprender y, por último, otro significado dice relación con la idea de toma de conciencia y metacognición (Quilaqueo, 2005; 2007).

${ }^{10}$ Esta situación puede ser equivalente a dificultades económicas y sociales, ya que los saberes educativos mapuches presentan una racionalidad que ha permitido al pueblo desarrollarse como tal y vivir en comunidades territoriales, en comunidades consanguíneas y en familias (Da Silveira et al., 2002); es decir, se trata de una proyección social con conocimientos culturales propios enseñados a niños, niñas y jóvenes.
} 
des, que simbolice el reconocimiento de la fundamentación y la explicación de sus acciones en el medio social (Ramírez, 2001). Desde una perspectiva política, la racionalidad es la adecuación de nuestras creencias a la realidad social, ya que proporciona una base segura al acierto de nuestras acciones y hace posible el consenso entre los individuos (Villoro, 1988). Por otro lado, desde el punto de vista de Habermas, se considera racional “... a la persona que interpreta sus necesidades a la luz de los estándares de valor aprendidos en su cultura, pero, sobre todo, cuando es capaz de adaptar una actitud reflexiva frente a los estándares de valor con que interpreta sus necesidades" (1987: 27).

En educación, la racionalidad, desde la visión de Giroux, es “....un conjunto específico de supuestos y prácticas sociales que median las relaciones entre un individuo o grupo y la sociedad amplia" $(2004: 218)^{11}$. A partir de esta definición, este autor plantea la necesidad de desarrollar " ... una nueva racionalidad y una nueva problemática para examinar la relación entre la escuela y la sociedad" (Giroux, 2004: 216). Esta nueva racionalidad permitiría reformular tanto la educación mapuche como la educación impartida por la escuela en su propio contexto, analizando el pasado, presente y futuro e indagando en las complejas relaciones entre el conocimiento y el poder dominante a partir de un pensamiento basado en el diálogo intercultural.

Subyacente a cualquier modo de racionalidad, tal como en la educación mapuche, se encuentra un conjunto de intereses que definen y califican cómo los sujetos se manifiestan ante los demás desde sus actividades familiares de sentido común, ya que "de hecho, existen dos tipos de racionalidad: una es específica de la actividad científica y la otra es propia de las actividades ordinarias de sentido común" (Coulon, 1995: 23). Este es un punto de vista epistemológico en el que los conocimientos, creencias, expectativas y tendencias que definen la racionalidad de la educación mapuche condicionan y son condicionados por las nuevas expectativas en las en que se plantean hoy día sus métodos educativos. En términos de Gasché (2001), esto se reconoce como doble racionalidad en las actividades socioculturales de las comunidades indígenas, “. $\ldots$ donde co-existen una lógica universal y una racionalidad vernácula, vinculada a las creencias" (Amado y Borzone, 2006: 12).

\footnotetext{
${ }^{11}$ Con respecto a la racionalidad en educación, Giroux se refiere a la educación ciudadana y plantea que si la racionalidad en la educación va a revitalizarse a sí misma en favor de la creación de una sociedad más digna y más justa, tendrá que liberarse de la carga de su propia historia intelectual e ideológica.
} 
Como enfoque epistemológico, los saberes educativos mapuches presentados en este trabajo se analizan basándose en la indagación con los mismos individuos, que se consideran simultáneamente como objeto y sujeto de sus relaciones sociales, con el fin de explicar las conductas colectivas relacionadas con dichos saberes a partir de la memoria social (Halbwachs, 1970); Le Goff, 1991; Sabourin, 1997; Quilaqueo, 1994 y 2005; Montesperelli, 2004) ${ }^{12}$. Los kimches encuentran en la memoria social, a partir de lo que ellos denominan kuifike zugu, los saberes educativos por segmentos, que cada uno utiliza de acuerdo con el tipo de conocimiento que se va a enseñar ${ }^{13}$. En síntesis, la memoria se vuelve colectiva e intersubjetiva al ser compartida, ya que va más allá de su objetivación (Berger \& Luckman, 1995; Montesperelli, 2004). Por otro lado, la desigualdad de oportunidades surge como crítica epistemológica (Van Haecht, 1999) con respecto al desarrollo de los saberes mapuches en el medio escolar, porque los afectados se ven confinados a la vida intrafamiliar e intracomunidad; lo que limita sus oportunidades de participación de las personas en las actividades laborales, culturales y sociales del país. Por su parte, Lahire (2008) plantea que son condiciones históricas que pueden transformarse en desigualdad social o cultural. En consecuencia, se trata de iniciar una explicación fundada en el paradigma de la identidad cultural (Bajoit, 2003).

Desde una perspectiva filosófica, para Foucault, "un saber es aquello de lo que se puede hablar en una práctica discursiva..." (1997: 306), mientras que para Lyotard (1994), refiriéndose al saber científico, éste no es todo el saber, ya que siempre ha estado en excedencia, en competencia, en conflicto con otro tipo de saber y, para resumir, este autor lo denomina saber narrativo. Ogien, por su parte, conceptualiza el saber como saber de sentido común y como conocimiento corriente, puesto que son “... dos nociones que la sociología emplea a menudo como si fueran análogas” (2007: 96).

${ }^{12} \mathrm{Al}$ respecto, se consideran los siguientes aspectos de la memoria social mapuche: la continuidad y discontinuidad de los valores educativos, las regularidades sociales, los cambios sociales y los problemas conceptuales que supone la rearticulación de los diversos hechos sociales una vez descritos.

${ }^{13}$ La memoria social ha sido la fuente principal de los conocimientos y de la identidad sociocultural mapuche. En expresiones de Groshens (1986) aparece como un elemento indispensable en la generación de la identidad de una colectividad y, para la educación mapuche, es el espacio donde se pueden encontrar esquemas reguladores. A partir de la condición social de los kimches, los principales elementos constitutivos de la memoria social de la educación mapuche se encuentran en contenidos como el zugu o tema en castellano, en contenidos procedimentales como el gülam o formas de enseñar mediante consejos y en contenidos actitudinales como, por ejemplo, el hecho de saber escuchar y saber respetar (Quilaqueo, 2006). 
En educación Zamorano (2005) considera la relación entre saber y representación, la asocia con la didáctica señalando que “... el conocimiento es el producto del acto de la ciencia y el saber, como una forma de gramática sobre la ciencia, que explica su origen, objetos y procedimientos" (2005: 61). Todos estos conceptos subyacen en el desarrollo de la investigación y de ellos derivan los resultados que se presentan en este artículo.

\section{METODOLOGÍA}

En primer lugar, en la investigación se utilizó una metodología cualitativa, ya que de acuerdo con Ruiz (1996) este enfoque obliga a tener una visión global del fenómeno que se va a estudiar, donde cada objeto de investigación puede ser entendido como un texto que se concibe dentro de un contexto y ambos deben ser abordados en su totalidad. Asimismo, se incentiva a no perder contacto con la realidad inmediata, puesto que la proximidad es un requisito indispensable. En segundo lugar, el diseño de este estudio se apoyó en la Teoría Fundamentada, que es definida por Strauss y Corbin como "... una teoría derivada de los datos recopilados de manera sistemática y analizados por medio de un proceso de investigación" (2002: 13) ${ }^{14}$. En tercer lugar, como punto de partida y núcleo temático, se consideraron los saberes educativos mapuches a partir de una muestra de kimches.

En consecuencia, el corpus está compuesto por el discurso de 30 kimches de las territorialidades Lafkenche, Naqche, Pehuenche y Wenteche de la Región de la Araucanía. Los kimches entrevistados pertenecen a las comunidades de Huapi, Traiguén, Trapilhue, Paliwepillán, Sahuelhue, Cumcumñaqui y Huentelar. Para la recopilación de los datos se utilizó la técnica de la entrevista semiestructurada, cuya primera parte se centró en la percepción global del entrevistado sobre las características de la educación mapuche. La segunda parte, en cambio, se centró en descubrir las experiencias personales de los kimches como expertos de la educación mapuche ${ }^{15}$. Asimismo,

\footnotetext{
${ }^{14}$ Con el fin de comprender la racionalidad de los saberes educativos mapuches, es necesario elaborar un cuerpo conceptual que se articule a través de proposiciones lógicas que permitan la construcción de una teoría. En este sentido, la conceptualización resultante del trabajo con la Teoría Fundamentada va más allá de un método descriptivo y sus limitaciones asociadas a la fiabilidad y generalización (Trinidad, Carrero y Soriano, 2006).

${ }^{15}$ Para la selección de los kimches de la muestra, se consideró a los que, por su conocimiento de la educación mapuche, demostraron ser los más idóneos y representativos. Asimismo, se consideró la información más relevante para el concepto o teoría buscada que el número o la selección al azar.
} 
el argumento metodológico para la aplicación de las entrevistas se apoyó en la saturación empírica de contenido (Valle, 1997; Pires, 1997). Por último, dichas entrevistas fueron transliteradas al castellano formal.

Se trabajó con 15 hombres y 15 mujeres kimches residentes de la Región de la Araucanía, cuyas edades fluctuaban entre los 45 y los 85 años y que, en su mayoría, tenían cónyuges mapuches. El 64\% de ellos había completado, al menos, cuatro años de enseñanza básica; un 25\% había recibido una escolarización básica incompleta y un $12 \%$ no había asistido a la escuela. Más de la mitad de la muestra (21) había sido miembro de organizaciones mapuches y cuatro de ellos habían desempeñado roles de liderazgo. El estatus económico de la muestra es bajo y medio bajo ${ }^{16}$.

En el análisis se consideró la lógica de construcción de conocimiento que se manifiesta en el discurso en mapunzugun de los kimches (Quilaqueo et al., 2004). Además, como estudio de problemas sociales relevantes, se consideró una postura crítica e interdisciplinaria, ya que existe una relación entre el discurso y las comunidades en un contexto socioeducativo (Colom, $1992)^{17}$. Al mismo tiempo, puede ayudar a que se aborden todos los niveles y dimensiones del discurso. Finalmente, se descubrió lo implícito, lo que estaba escondido y que, por algún motivo, no era inmediatamente obvio en las relaciones de dominación discursiva o de sus ideologías subyacentes $\left(\right.$ Wodak, 2003) ${ }^{18}$. Como elemento articulador del proceso de codificación y categorización teórica ${ }^{19}$, se consideró el Método Comparativo Constante y, asociado a éste, se consideró también un segundo elemento que es la Saturación Teórica ${ }^{20}$. Por su parte, el diseño cualitativo admite también cambios

\footnotetext{
${ }^{16}$ Las razones teóricas para trabajar con esta muestra fue el conocimiento educativo validado en la memoria social mapuche que los kimches conocen, ya que sus conocimientos permiten dar una interpretación adecuada sobre el saber educativo y, por último, al describir los principales saberes educativos, se apela a los valores desde la lógica cultural mapuche que subyace en el discurso de los kimches.

${ }_{17}$ Para controlar la calidad de la investigación se recurre a la triangulación propuesta por Denzin (1978).

${ }^{18}$ De acuerdo con esto, las categorías del saber educativo consideran otras dimensiones presentes en el discurso de los kimches, se trata de dimensiones que se sitúan en un continuum de propiedades valóricas presentes en la memoria social (Halbawchs, 1970; Sabourin, 1997).

${ }^{19}$ Este método es un procedimiento que sirve para interpretar textos, comparando una y otra vez de manera sistemática los códigos a medida que se van definiendo. Así, un investigador analiza datos para elaborar conceptos, al mismo tiempo que codifica, comparando ocurrencias específicas de los datos.

${ }^{20}$ Esto ocurre cuando las comparaciones no arrojan nuevas relaciones o nuevas propiedades de los datos. Uwe Flick (2004: 79) plantea que “... el muestreo e integración de material nuevo se acaba cuando la saturación teórica de una categoría o grupo de casos se ha alcanzado, es decir, cuando no surge ya nada nuevo".
} 
durante el transcurso de la investigación con el fin de acercarse lo más posible a la comprensión del fenómeno en cuestión (Ruiz Olabuénaga, 1996)²1.

\section{RESULTADOS}

Los códigos que han alcanzado la saturación dan como resultados las siguientes categorías: kimeltuwün (proceso y acción educativa mapuche), kimün (saberes y conocimientos) y prácticas socioculturales. El análisis se realizó a partir de los datos de cada código de las subcategorías y su frecuencia de uso, a partir del proceso de codificación teórica (Ver Tabla 1).

Tabla 1. Frecuencias de uso de los códigos de las subcategorías kimeltuwün, kimün y prácticas socioculturales.

\begin{tabular}{|l|c|l|l|l|c|}
\hline \multicolumn{2}{|c|}{ Kimeltuwün } & \multicolumn{2}{c|}{ Kimün } & \multicolumn{2}{c|}{ Prácticas socioculturales } \\
\hline Enseñanza Kimche & $26,8 \%$ & Küpan & $36,1 \%$ & Prácticas sociales & $21,6 \%$ \\
\hline Formación en la niñez & $45,2 \%$ & Tuwün & $24,2 \%$ & Ceremonias & $17,6 \%$ \\
\hline $\begin{array}{l}\text { Formas de construcción } \\
\text { conocimiento }\end{array}$ & $28,0 \%$ & Oficios & $39,7 \%$ & $\begin{array}{l}\text { Creencias en } \\
\text { günechen }\end{array}$ & $60,8 \%$ \\
\hline \multicolumn{1}{|c|}{ Total } & $100 \%$ & Total & $100 \%$ & Total & $100 \%$ \\
\hline
\end{tabular}

Fuente: Elaboración propia, mediante el software Atlas-ti 5.

La categoría kimeltuwün contiene los códigos conceptuales de: enseñanza kimche, que representa un 26,8\%; formación en la niñez, un 45,2\% y formas de construcción de conocimientos, un 28,0\%. En la categoría kimün, el küpan es parte de los contenidos enseñados y representa el 36,1\%; el tuwün, asociado al contenido del küpan, es parte de los principales contenidos enseñados y representa el 24,2\%; por último, el código de oficios enseñados representa el 39,7\%, porque contiene y explica los dos contenidos de los códigos de küpan y tuwün. La categoría prácticas socioculturales es consecuencia, principalmente, del código de creencias en günechen, que es el más

${ }^{21}$ Ruiz Olabuénaga (2003) señala que se intenta comprender en su totalidad el objeto de estudio, nunca fragmentado o aislado del contexto. Es necesario comprender e incorporar el conocimiento de los informantes y lograr que éstos hablen por sí mismos para que se acerquen a su experiencia particular desde los significados y la visión del mundo que poseen. 
frecuente y alcanza un $60,8 \%$; las prácticas sociales, por su parte, alcanzan un $21,6 \%$ y las ceremonias, el $17,6 \%$.

\section{CATEGORÍA KIMELTUWÜN}

Kimeltuwün se define como acción educativa que implica un proceso de aprendizaje y enseñanza entre dos o más personas que tienen la intención de abordar un contenido. Éste sintetiza el proceso educativo mapuche que aborda aspectos tales como quiénes aprenden, cómo y qué se enseña considerando la edad del niño o de la niña. Los resultados muestran que este proceso incluye principalmente elementos valóricos como la responsabilidad familiar en la enseñanza y la exclusividad de los padres y abuelos en la entrega de conocimientos cotidianos para la formación de los niños y jóvenes. Los conocimientos son de tipo práctico, acompañados de consejos que abordan cuestiones valóricas, míticas e históricas ancestrales. De la categoría kimeltuwün se desprenden tres conceptos: formación en la niñez, formas de construcción de conocimientos y enseñanza kimche. En esta categoría, el concepto de formación en la niñez es el que se presenta con mayor frecuencia y alcanza un $45,2 \%$. Se define como la entrega constante de consejos, donde un tipo de conversación denominado güxam constituye la base del método de enseñanza. Ante la pregunta ¿qué temas se abordan para que los niños aprendan?, un kimche indica que "se le habla en mapunzugun, se tratan temas acerca de la convivencia: vivir bien, cuál es la forma correcta de invocar a günechen. Eso es lo que se enseña..." (Entrevista 28). Esto demuestra que la base de la enseñanza es un contenido de la vida cotidiana y simbólico espiritual sobre günechen. En cuanto a cómo se entregaba la formación de los niños y niñas en épocas pasadas, se señala que "... se le comparaba con otro joven o niño de su misma edad o de más edad para que tuviera conciencia de la diferencia con el otro y fuera elegido para ser guiado por un kimche que tenía la sabiduría" (Entrevista 22).

En cuanto al concepto construcción de conocimientos, su frecuencia es de un $28 \%$. En este punto se explicitan estrategias y métodos utilizados, principalmente, la comprensión. Comprensión e interpretación es lo que los kimches utilizan para explicar la realidad de la vida cotidiana y la construcción de conocimientos de los niños. En este sentido, la interpretación de sueños, denominada peuma en lengua mapuche, funciona como fuente de conocimiento. $\mathrm{Al}$ respecto, una mujer que se casó y se integró a una familia de una determinada comunidad y que no conocía la forma de enseñar a sus hijos en la tradición mapuche explica su experiencia de aprendizaje 
mediante sueños, especificando que “... en el sueño le dicen a uno cómo realizar un trabajo,... un consejo a los hijos. De esta manera, esa persona sabrá cómo hacer...". Esta mujer explica su situación vivida señalando que “... llegué a sufrir harto y se reían de mí, me decían que era señora wigka $a^{22}$. Yo lloraba mucho y me sentía muy mal, pero una noche soñé y, así, aprendí y ahora conozco las costumbres mapuches como hacer gülam, por ejemplo" (Entrevista 10).

El método de enseñanza que desarrolló la entrevistada es conocido como gülam y corresponde a una forma de consejo formativo de carácter temático entregado durante la niñez y la adolescencia. Se puede observar que los padres y los familiares más cercanos intervienen mediante este método en la formación de los niños y se señala que “... el hombre y la mujer, la madre o el padre tienen la misma obligación de entregar el gülam...”. Sin embargo, se recalca en las explicaciones de los kimches que "... esto antes también era relativo, puesto que si en una familia uno de los padres tenía más conocimiento, esta persona era la encargada de dar gülam..." (Entrevista 19). Es decir, se buscaba formar a los niños con la persona que poseía mayores conocimientos socioculturales del grupo familiar y de la comunidad.

Por su parte, el concepto de enseñanza kimche alcanza una frecuencia de un $26,8 \%$ y se define como la comprensión e interpretación del discurso educativo de los kimches, que ellos denominan pensamiento educativo mapuche. El pensamiento educativo consiste en un razonamiento que define cómo construir los conocimientos con los niños, niñas y jóvenes. A este procedimiento, uno de los kimches lo denomina pensaduría, que se entiende como la manera que él tiene de representar la racionalidad de lo que explica, porque engloba aspectos sociales y culturales en el contexto de las actuales relaciones interculturales e interétnicas. Esta reflexión del kimche se refiere esencialmente al aprendizaje que puede lograr una persona sobre hechos que se comunican a partir de la memoria social como el kuifike $z u g u$. En consecuencia, se afirma que sólo puede enseñar el que aprende. De aquí se desprende que, para aprender, la persona debe observar, escuchar y preguntar. Los kimches, en su mayoría ( $80 \%$ de la muestra), afirman que “... el verdadero kimche puede llegar a la esencia del conocimiento mapuche, puesto que conoce la tradición para reconstruir el conocimiento recurriendo a etapas retrospectivas de tiempo pasado o kuifi" (Entrevista 10).

Entonces, desde la perspectiva de la enseñanza de los kimches, se pueden reconocer aspectos vitales que reflejan la identidad mapuche, tales como el

\footnotetext{
${ }^{22}$ El término wigka es utilizado, hoy día, para referirse al chileno de origen español.
} 
concepto mapunche $e^{23}$. De esta manera, de acuerdo con el tipo de relación que se establece con el medio social, natural y espiritual, el término mapunche se refiere a cómo un mapuche asume su identidad territorial y familiar, mientras que el término mapuche etimológicamente significa gente de la tierra o gente del lugar. En síntesis, esta categoría explica las tres grandes áreas de conocimientos que utilizan los kimches para enseñar: medio social, medio natural y aspectos espirituales. Así, se hace explícito, por ejemplo, el comportamiento de las personas como un aspecto vital de los mapuches y se explica como el hecho de "saber comportarse" en relación con las tres áreas señaladas. Por lo tanto, la finalidad de este tipo de enseñanza es realizar buenas reflexiones como ejemplo ante los demás, puesto que “... de esta forma hay una buena comunicación entre las personas, se invoca a günechen, se entregan buenas ideas, se hace un buen uso del lenguaje y hay una buena actitud para tener un comportamiento adecuado. Eso decían nuestros antepasados..." (Entrevista 30).

\section{CATEGORÍA KIMÜN}

Kimün se revela, en general, como el saber mapuche en los distintos campos del desarrollo humano (social, cultural, económico, entre otros). No obstante, para el objetivo de este estudio, el kimün se consideró solamente como parte de los saberes educativos para la formación de persona y de los oficios. De lo anterior se desprende que los distintos saberes se pueden clasificar como saberes relacionados con la naturaleza, la sociedad, la lengua, la salud, las formas de organizar la comunidad, las condiciones y los pasos (métodos) para formar a una machi (persona encargada de la salud en una comunidad), a una partera, etc. Desde un punto de vista más práctico y cotidiano, se encuentran, por ejemplo, todos los saberes relacionados con los oficios como la textilería y la agricultura que alcanzan una frecuencia de un $39,7 \%$. Entre los saberes educativos más relevantes en la formación de

${ }^{23}$ El concepto mapunche es citado en los textos de Campos Menchaca (1972: 29), cuando señala que “... don Alonso de Ercilla inventó la palabra araucano, tomándola del nombre del fuerte; la palabra pasó después a designar la raza de los mapuches, su idioma y su territorio, tanto que da lo mismo ahora decir mapuche o araucano. Los indígenas despreciaron esa denominación por provenir de los huincas y siguen llamándose mapuche o mapunche". También, se aclara que los términos mapuche y mapunche, “... son dos denominaciones que los mapuches han utilizado para referirse a sí mismos, de acuerdo con la manera de relacionarse, tomando en cuenta la ascendencia parental y su relación con el entorno natural, social, espiritual y la explicación a través del lenguaje respecto del territorio que ocupan" (Quilaqueo, 2005: 25). 
personas se encuentran los conceptos de la ascendencia familiar y territorial de cada persona, denominados küpan y tuwün respectivamente. Estos dos conceptos sintetizan la construcción del conocimiento para los niños $\mathrm{y}$, al mismo tiempo, se consideran hitos familiares para reconocerse como mapuche y mapunche, según sea el grado de conocimiento sociocultural de la persona y la territorialidad a la cual pertenezca.

El concepto küpan, que alcanza un $36,1 \%$ de frecuencia, distingue los aspectos de ascendencia familiar paterna más importantes de la categoría kimün. Se señala que es un conocimiento básico que debe poseer un sujeto sobre su ascendencia familiar. De esta forma, cuando a los niños se les enseña mediante un gülam (tipo de consejo), se les instruye sobre los distintos grados de parentesco y los distintos tipos de $a z$ o rasgos de personalidad de sus familiares, por ejemplo, “... en un gülam se le dice quiénes eran sus tíos y abuelos, porque si uno no hace eso después los niños no saben quiénes son sus familiares... enseñándole las principales características de ascendencia. Es decir, como a mí me enseñaron..." (Entrevista 06).

Por su parte, el concepto tuwün, que alcanza un $24,2 \%$ de frecuencia, se refiere, en términos más generales, al origen materno-territorial de un individuo; sin embargo, implica muchos aspectos más del territorio en el cual se desenvuelve el sujeto. Según los kimches, tuwün se refiere al conocimiento del origen geográfico del individuo con respecto a sus antepasados. Un ejemplo en el uso de este concepto se observa en el siguiente discurso: “... sí, como lo ha dicho el peñi [hermano] que anda con Usted. Usted escuchó que mencionó de donde provenía él. Ha dicho que viene del lado del mar y nosotros diremos vino una visita que proviene del lado del mar: es lafkenche. Esa es la información que voy a tener ahora" (Entrevista 13). Es decir, lo que el kimche recalca es el conocimiento territorial que él tiene de la comunidad lafkenche o comunidad territorial del lado del Océano Pacífico.

\section{CATEGORÍA PRÁCTICAS SOCIOCULTURALES}

Mediante las prácticas socioculturales se expresa que en cada persona subyace la creencia en un espíritu superior denominado günechen. Entre los kimches de la muestra de este estudio, la referencia a günechen es mayoritariamente frecuente y alcanza el 60,8\%. A partir de esto se puede distinguir un aspecto que es más religioso y otro que tiene un carácter más social. El concepto de religiosidad que se manifiesta se desprende de los principales ceremoniales y su frecuencia en el testimonio de los kimches es de 
un $17,6 \%$. Entre estas manifestaciones ceremoniales se destaca el gijatun (ceremonia sociorreligiosa) y las oraciones de cada participante tales como el gijatu. Asimismo, se destaca el awün (saludo ceremonial y respeto a las personas) y el misawün (comida compartida en una reunión sociocultural). La religiosidad se expresa como gratitud y petición en el gijatun y se señala, por ejemplo: "Gran padre te pedimos que nos libres de enfermedades a todas nuestras familias y también a nuestros animales, te pedimos por nuestro futuro... Te agradecemos por el agua que tu das cada día, por el sol que nos siga iluminando...” (Entrevista 24).

Entre las características de las prácticas sociales, cuya frecuencia alcanza un $21,6 \%$, se destaca el conocimiento construido para desarrollar los discursos wewpin, como un espacio para construir conocimiento. El contenido se refiere al conocimiento ancestral de cada familia y comunidad y se indica que el wewpin se realizaba “... sobre temas elegidos para la conversación en grupo o entre dos personas... Allí se producía una forma de interrogación entre los kimches, se preguntaban recíprocamente con respecto a la información o conocimientos que tenía cada uno sobre el tema elegido..." (Entrevista 16). Aquí se observa que el discurso se hace en tiempo pretérito para explicar lo que es el wewpin cuando se específica que “... eso era lo que hacía la gente antes, la gente que sabía, la gente que hacía gülam, por ejemplo, la gente más anciana, ellos eran los que llevaban adelante las conversaciones" (Entrevista 27). Con este argumento se pone énfasis en el carácter temático del weupin y en la manera de construir conocimientos recurriendo al tiempo pasado o, como lo señala Morin (2002), mediante el reencuentro con el pasado es que un grupo humano encuentra la energía para enfrentar su presente y preparar su futuro. Además, se señala que el weupin sólo lo realizaban las personas que tenían el conocimiento mediante la estrategia educativa gülam (Quilaqueo, 2006). En los testimonios se observa que, cuando se refieren a la gente más anciana, en realidad se están refiriendo a un kimche, persona que ha logrado ser reconocida como tal por las familias de una comunidad.

\section{DISCUSIÓN}

En las últimas décadas del siglo pasado y, desde un enfoque no mapuche, se realizaron estudios centrados en la descripción etnográfica (Bradford, 1976) o sociohistórica (Bengoa, 1987) y en aspectos relacionados con creencias y relatos sobre mitos mapuches, desde una perspectiva lingüística 
(Kuramochi y Nass, 1988); sin embargo, estos trabajos, en general, no vinculan la lógica universal con la racionalidad que subyace en el saber educativo mapuche. Considerando los estudios de Gasché (2004), al referirse a un modelo sintáctico de cultura, señala que existe una racionalidad oculta en las creencias que originan la realización de ciertos actos y discursos míticos (ver también, Amado y Borzone, 2006). En el caso mapuche, son del mismo modo actos y discursos míticos los que están vinculados a creencias e ideas de orden universal que configuran la existencia de una doble racionalidad del actual conocimiento educativo. Esto se refiere a prácticas de la vida cotidiana, que están relacionadas con aspectos simbólicos sobre günechen y conocimientos universales de tipo biológico, físico, geográfico, o contenidos como los conceptos de tuwün y küpan.

En este estudio, se puede constatar, en general, que hasta ahora la literatura acerca de los mapuches no da cuenta de un marco conceptual de saberes educativos y culturales mapuches pertinentes para que sean incorporados al currículum escolar. Desde esta perspectiva, la categorización realizada en este trabajo puede servir como orientación para construir un marco teórico que haga explícita la relación del saber o kimün con la lógica del pensamiento cultural mapuche y no mapuche, cuyo objetivo podría ser, por ejemplo, un enfoque pedagógico intercultural (Quilaqueo, 2007). De esta manera, se podría superar el problema del desconocimiento del saber educativo mapuche a partir de una nueva forma de relacionarse con el saber (Charlot, 1997; Beillerot, 1998). El objetivo final es incorporar ambas lógicas de pensamiento para plasmarlas en un currículum intercultural de escuelas ubicadas en contextos mapuches (Quilaqueo, Quintriqueo y Cárdenas, 2005). Es decir, es necesario contar con un currículum integrador que incorpore ambas lógicas de pensamiento, puesto que "... si desde la escuela no se valoriza lo que cada uno es y sabe, los gustos e intereses personales, se estarán negando la identidad y el derecho a ser de cada uno" (Sagastizabal, 2006: 87). En consecuencia, surge la necesidad e importancia de reconocer y valorizar la lengua mapunzugun, ya que permite conocer la complejidad de las expresiones socioculturales de la identidad sociocultural mapuche. Esta idea supone replantear el estado del conocimiento educativo propuesto en un currículo monocultural que, de acuerdo con Morin (2002 y 2004), tiende a la simplificación y fragmentación del conocimiento, lo que causa incomprensión de la realidad e identidad de los sujetos y no permite dar respuestas a la complejidad e incertidumbre de la vida. En términos de interculturalidad, todo esto exige una confrontación permanente de distintos puntos de vistas (Abdallah-Pretcheille, 1996), porque la inter- 
culturalidad no se refiere a la incorporación del uno en lo del otro, como en lo religioso, moral o ético, sino que busca más bien la transfiguración de lo propio y de lo ajeno sobre la base de lo común y en pos de la creación de un espacio común determinado por la convivencia (Fornet-Betancourt, 2001).

De este modo, las categorías conceptuales pertinentes a una lógica de pensamiento occidental de la educación podrían incorporar, a partir de los saberes y conocimiento mapuches que corresponden a contenidos conceptuales, procedimentales y actitudinales (Bixio, 1997; Quilaqueo et al., 2004), saberes tales como el küpan y el tuwün, y valores como respetar y saber escuchar. Asimismo, se podría incorporar el procedimiento metodológico gülam, que se apoya en una lógica que le permite a la familia mapuche mostrar a sus hijos la entrega del conocimiento ancestral mediante consejos. Para entregar estos consejos se recurre a procedimientos simbólicos que parecen irracionales (Wallerstein, 2005) para los no mapuches y, actualmente, también para muchos jóvenes mapuches no socializados en su cultura, particularmente el uso del kuifike zugu. Este procedimiento simbólico implica recurrir a la memoria social de la familia, de la comunidad consanguínea y de la comunidad territorial, desde el saber ancestral con las continuidades y discontinuidades que presenta. Este tipo de educación, sustentado en la memoria social, presenta dificultades a las nuevas generaciones, porque no poseen un buen conocimiento de la lengua mapunzugun, lo que influye negativamente en la comprensión de los valores educativos y en las regularidades del uso de los métodos sociales de formación. En consecuencia, los cambios sociales y culturales que se observan crean problemas debido al desconocimiento de los diversos hechos sociales descritos a partir de la memoria social (Sabourin, 1997; Montesperelli, 2004; Quilaqueo, 1994 y 2005).

Desde la perspectiva de los kimches, la problemática de la racionalidad de los conocimientos educativos y los principios que han guiado la formación de los niños y niñas les ha permitido, de acuerdo con las ideas de Wallerstein (2005), desarrollar su cultura y un tipo de relación social entre familias y comunidades. En la educación mapuche, la racionalidad se presenta como lo que se ha denominado principios que guían la formación de la persona. Desde un punto de vista evaluativo de la formación, se reconoce a la persona que actúa con el grupo parental, pero esto se sintetiza en el principio kim che geal, que guía la formación de la persona sustentada en el conocimiento que trasciende lo social y cultural, y que se resume en el respeto a günechen, categoría que en este trabajo ha alcanzado la mayor frecuencia, un $60,8 \%$. Todo esto permite plantear la hipótesis de la doble 
racionalidad de los saberes educativos mapuches, que se sustenta en la idea de hombre-naturaleza y aspectos simbólicos relacionados con günechen, ya que günechen sería lo que le da sentido y utilidad a la educación y formación de las personas, es decir, lo que permite racionalizar los saberes. Considerando lo expresado por los kimches, la doble racionalidad se puede extender a lo que se realiza en las escuelas situadas en los nuevos contextos de vida mapuche. En síntesis, los principios descritos y la expresión pensaduría, planteada por un kimche, corresponden tanto a la explicación de la racionalidad de la educación mapuche a partir de la memoria social ancestral como a la explicación actual del comportamiento a partir de la educación mapuche de hoy.

\section{CONCLUSIÓN}

Los resultados obtenidos en este estudio revelan que la educación que se utiliza para formar a niños, niñas y jóvenes mapuches entrega contenidos a través de métodos educativos propios para tratar los aspectos sociales, culturales, religiosos y naturales. En particular, se identifican contenidos de saberes específicos de la educación familiar como, por ejemplo, el küpan y el tuwün. A partir de estos contenidos, esta investigación ha develado un primer acercamiento sobre la construcción de conocimientos educativos desde la lógica mapuche.

En la lógica de construcción de los conocimientos educativos mapuches, primero, se pone en evidencia el rol que cumplen los kimches, ya que son los sujetos portadores de los saberes culturales y educativos, porque su sabiduría y conocimientos permiten explicar las conductas de los niños, niñas y jóvenes en el medio escolar. Segundo, se evidencia que la memoria social de las familias (memoria social que incluye las relaciones interétnicas con los no mapuches) es la base para comprender la doble racionalidad que presentan, hoy día, los niños, niñas y jóvenes en sus participaciones en contextos externos a su medio familiar y comunitario, principalmente, al comenzar la escolarización.

Considerando la problemática de la racionalidad de los saberes educativos mapuches y la necesidad de su incorporación al currículum escolar, es necesario desarrollar nuevos enfoques pedagógicos y didácticos para la formación de los alumnos de origen mapuche y no mapuche, ya que mediante una mejor relación con los saberes se pueden cambiar las interacciones entre los mapuches y no mapuches. De esta manera, se puede también re- 
conocer la doble racionalidad que los niños y niñas mapuches manifiestan en sus relaciones con los saberes, para evitar el choque psicosocial que se produce mediante la escolarización descontextualizada. Esto significa que la investigación educativa debe problematizar con la finalidad de estudiar las situaciones de doble racionalidad que los niños y niñas mapuches presentan. Para ello, la hipótesis del enfoque pedagógico intercultural permite considerar las distintas variables que intervienen, tanto en la formación mapuche como en la formación general que entrega la escuela. Además, se debe considerar la matriz cultural occidental que, a su vez, es intercultural, y que, al mismo tiempo, está en función de las situaciones interpersonales e intergrupales en las cuales se presenta. En consecuencia, la investigación se debe plantear a partir de un enfoque intercultural histórico y estratégico como premisa fundamental para una comprensión más singular de la desigualdad de oportunidades de la escolarización actual.

Por su parte, en la escuela todavía hay una dominación cultural impulsada por la castellanización, que genera la desigualdad de oportunidades para los alumnos mapuches frente a los saberes no mapuches y, del mismo modo, una desigualdad para los alumnos no mapuches frente a los saberes ancestrales mapuches. Así, la monoculturalidad de la escuela, como condición histórica, no logra superarse, aun cuando se propone la interculturalidad como reflexión crítica que busca la transformación de la cultura propia y la ajena, sobre la base de una nueva racionalidad educativa que apunte a la construcción de espacios comunes de convivencia.

Finalmente, en la indagación con los kimches, ellos hacen hincapié en los aspectos éticos que implica la oralidad de sus saberes educativos, subrayando que el verdadero sabio no puede transgredirlos. El aspecto ético al cual se refieren se apoya en el argumento de la creencia profunda en la palabra como un don sobrenatural entregado por günechen, que involucra deberes y obligaciones, y que este don, como fuerza sobrenatural, es logrado por quienes cultivan el conocimiento y la lengua mapunzugun (se refieren a la expresión zapin, utilizada como verbo para señalar la acción de cultivar los conocimientos). No obstante, al mismo tiempo, son conscientes de que sus conocimientos les imponen deberes frente a günechen, a sus familiares $y$, en general, a la comunidad mapuche y no mapuche. Entre los deberes, se señalan varias obligaciones: demostrar razonamiento y conocimiento en sus relaciones; guardar respeto de los valores morales y denunciar las desviaciones; cuidar el uso del lenguaje (del mapunzugun y hoy día también del castellano); decir la verdad; y entregar las informaciones que permitan enseñar mediante la base de la memoria social para transmitirla a las fu- 
turas generaciones. De estas obligaciones se desprenden varias funciones para los kimches como formadores. Así, ellos señalan estar conscientes de ser conocedores de los valores morales y espirituales, de la historia de las comunidades como memoria colectiva, entre otros aspectos. En síntesis, la memoria social mapuche es la biblioteca donde se conserva la información más importante, principalmente los hechos que han marcado la historia de la formación de persona positivamente o negativamente como efecto de la escolarización de la población mapuche.

\section{REFERENCIAS}

Abdallah-Pretceille, M. (1996). Vers une pédagogie interculturelle. Paris: Anthropos. Amado, B.; Borzone, A.(2006). "El concepto de la doble racionalidad en la educación". Revista del Instituto de Investigaciones en Ciencias de la Educación, Facultad de Filosofía y Letras, Universidad de Buenos Aires, pp. 20-26.

Augusta, F. (1903). Lecturas araucanas. Temuco, Chile: Editorial Kushe.

Bajoit, G. (2003). Todo cambia: Análisis sociológico del cambio social y cultural en las sociedades contemporáneas. Santiago de Chile: Lom Ediciones.

Beillerot, J. (1998). "La relación con el saber: una noción en formación". En: Jacky Beillerot, Blanchard, Claudine y Mosconi, Nicole, Saber y relación con el saber. Buenos Aires: Editorial Paidós SAICF.

Bengoa, J. (1987). Historia del pueblo mapuche. Santiago de Chile: Ediciones Sur. Colección Estudios Históricos.

Berger, P.; Luckmann, T. (1995). La construcción social de la realidad. Buenos Aires: Amorrortu.

Bixio, C. (1997). Contenidos procedimentales. Los procedimientos: su enseñanza, aprendizaje y evaluación. Rosario, Argentina: Homo Sapiens Ediciones.

Bradford, M. (1976). "El sistema de valores del mapuche." En: Dillehay, Tom (compilador), Estudios antropológicos sobre los mapuches de Chile sur-central. Temuco, Chile: Pontificia Universidad Católica de Chile, Sede Regional.

Campos, M. (1972). Por senderos araucanos. Buenos Aires: Editorial Francisco de Aguirre.

Catrileo, M. (1995). Diccionario Lingüístico-etnográfico de la lengua mapuche. Santiago de Chile: Editorial Andrés Bello.

Charlot, B. (1997). Du rapport au savoir. Élements pour une théorie. Paris: Anthropos.

Colom, A. (1992). Modelos de Intervención Socioeducativa. Madrid: Nancea S. A. de Ediciones.

CONADI, Corporación Nacional de Desarrollo Indígena (1993). Ley Indígena. (Ley No 19.253 D. of. $\left.5^{\circ} 10^{\circ} 1993\right)$. Temuco: CONADI.

Coulon, A. (1995). Etnometodología y educación. Barcelona: Ediciones Paidós Ibérica, S. A. 
Da Silveira, Y.; Maheux, G.; Simard, D.; Quilaqueo, D. (2002). “Construction de savoirs professionnels et échanges interculturels en contexte de formation des enseignantes inuit et mapuche". Collectif interculturel. La revue de l'Institut de Recherche et de Formation Interculturelles de Québec, V., No 2, 167-185.

Denzin, N. (1991). Interpretative Interaccionism. (4a ed.) California. United States of America: Editorial Sage.

Erize, E. (1960). Diccionario comentado mapuche-español: Araucano, pehuenche, pampa, picunche, ranculche y huilliche. Buenos Aires, Argentina: Cuadernos del Sur. Instituto de Humanidades. Universidad Nacional del Sur.

Fairclough, N. (1995). Critical Discourse Analysis: the Critical Study of Language. London: Longman.

Flick, U. (2004). Introducción a la investigación cualitativa. Madrid: Ediciones Morata, S. L.

Fornet-Betancourt, R. (2001). Filosofía e interculturalidad en América Latina. Intento de introducción no filosófica. En: Heise M. (ed.) Interculturalidad. Creación de un concepto y desarrollo de una actitud (pp. 63-73). Lima: Inversiones Hathuey S.A.C.

Foucault, M. (1997). La arqueología del saber (18 ${ }^{\text {a }}$ ed.). México DF: Siglo XXI Editores.

Gasché, J. (2001). "El reto de una educación indígena amazónica”. Cultura y Educación, Vol. 13, 59-72.

— (2004). "Niños, maestros, comuneros y escritos antropológicos como fuente de contenidos indígenas escolares y la actividad como punto de partida de los procesos pedagógicos interculturales: un modelo sintáctico de cultura”. [On line]. En: Red internacional de estudios interculturales, Pontificia Universidad Católica del Perú. Disponible en: http://red.pucp.edu.pe/ ridei/wp-content/uploads/biblioteca/ninos_maestros_comuneros_modelo_sintactico_de_cultura.pdf [Consulta: 18.05.10].

Giroux, H. (2004). Teoría y resistencia en educación (6 a edición). Buenos Aires: Editorial Siglo XXI.

Groshens, M.-C. (1986). Production d'identité e mémoire collective. En: Tap, Pierre, Identités collectives et changements sociaux (pp. 149-151). Toulouse: Privat.

Habermas, J. (1987). Teoría de la acción comunicativa, tomos I y II. Madrid: Editorial Taurus.

Halbwachs, M. (1970). Morphologie sociale. París: Armand Colin.

Housse, E. (1939). Une épopée indienne. Les Araucans du Chili. París: Librairie Plon.

Kuramochi, Y.; Nass, J. (1988). "Kamarrikún. Valoración de la tradición y causalidad". Actas de Lengua y Literatura Mapuche, № 3, 26-56.

Lahire, B. (2008). "Cultura escolar, desigualdades culturales y reproducción social". En: Tenti Fanfani, E. (compilador), Nuevos temas en la agenda de la política educativa. Buenos Aires: Siglo XXI Editores. 
Latcham, R. (1924). La organización social y las creencias religiosas de los antiguos araucanos. Santiago de Chile: Imprenta Cervantes.

Le Goff, J. (1991). El orden de la memoria, el tiempo como imaginario. España: Editorial Paidós.

Loncón, E. (1997). Construyendo una educación intercultural bilingüe mapuche. Propuestas y desafíos. Temuco: Sociedad Interdisciplinaria de Estudios y Desarrollo Social, SIEDES.

Lyotard, J.-F. (1994). La condición postmoderna. Madrid: Cátedra.

MINEDUC, Ministerio de Educación (2003). Orientaciones para la formulación de proyectos de mejoramiento educativo en el marco de la educación intercultural bilingüe. Santiago: Departamento de Educación Intercultural Bilingüe. Gobierno de Chile, División de Educación General.

Molina, J. I. (1778). Compendio de la historia geográfica, natural y civil del reyno de Chile. Traducción de Domingo Joseph. Madrid: Antonio de Sacha.

Montesperelli, P. (2004). Sociología de la memoria. Buenos Aires: Nueva Visión.

Morin, E. (2002). Los siete saberes necesarios para la educación del futuro. ( $3^{\mathrm{a}}$ reimpresión) Buenos Aires: Ediciones Nueva Visión.

- (2004). La mente bien ordenada. Repensar la reforma. Reformar el pensamiento. ( $6^{\mathrm{a}}$ ed.) Barcelona: Editorial Seix Barral.

Noggler, A. (1972). Cuatrocientos años de misión entre los Araucanos. Padre Las Casas, Temuco: Editorial San Francisco.

Ogien, A. (2008). Las formas sociales del pensamiento. La sociología después de Witgenstein. Buenos Aires: Nueva Visión.

OIT, Organización Internacional del Trabajo [1989] (1996). Convenio Nº 169 sobre Pueblos Indígenas y Tribales en países independientes. En: Revista América Indígena, Volumen LVIII, Nos 3-4, julio-diciembre, pp. 37-56.

Pires, A. (1997). "Échantillonnage et recherche qualitative: essais théorique et méthodologiques". En: Poupart, Jean et al., La recherche qualitative. Enjeux épistémologiques et méthodologiques (pp. 256-297). Montréal: Gaëtan Morin éditeur.

Quilaqueo, D. (1994). "Participation Traditionnelle et Participation Provoquée. Les nouvelles communautés mapuche de deux provinces d'Argentine: Neuquén et Río Negro". Mondes en Développement, de l'Institut de Mathématiques Appliquées ISMEA, tome 22, $\mathrm{N}^{\circ} 86,17-26$.

- (2005). "Educación Intercultural desde la teoría del control cultural en contexto de diversidad sociocultural mapuche". Cuadernos Interculturales, año 3, $\mathrm{N}^{\circ} 4$, enero-junio. Centro de Estudios Interculturales y del Patrimonio (CEIP). Universidad de Valparaíso, 37-50.

_ (2006). "Valores educativos mapuches para la formación de persona desde el discurso de kimches”. Estudios Pedagógicos XXXII, N² 2; Valdivia, 73-86.

(2007). "Saberes y conocimientos indígenas en la formación de profesores de educación intercultural”. Educar em Revista, No 29, 223-239.

_ (2010). "Racionalidad de los saberes educativos mapuche apoyada en 
la memoria social de los kimches". En: Quilaqueo, Daniel; Fernández, César; Quintriqueo, Segundo, Interculturalidad en contexto mapuche (pp. 55-82). Neuquén, Argentina: Editorial Educo.

Quilaqueo, D.; Quintriqueo, S.; Cárdenas, P. (2005). Educación, currículum e interculturalidad. Elementos sobre formación de profesores en contexto mapuche. Santiago: Frasis editores - Facultad de Educación, Universidad Católica de Temuco.

Quilaqueo, D.; Quintriqueo, S.; Catriquir, D.; Llanquinao, G. (2004). “Kimeltuwün mew amukey ta zugu. Una didáctica para abordar conocimientos mapunche en el proceso de formación inicial en educación intercultural". En: Grupo de Investigación, Cuatro estudios para mejorar la formación inicial docente (pp. 53-148). Temuco, Chile: Facultad de Educación. Universidad Católica de Temuco.

Quintriqueo, S. (2005). "Implicancias de la escolarización en la construcción de la identidad cultural de alumnos mapuche en el medio escolar de la IX Región”. En: Quilaqueo, Daniel; Quintriqueo, Segundo; Cárdenas, Prosperino, Educación, currículum e interculturalidad. Elementos sobre formación de profesores en contexto mapuche (pp. 191-248). Facultad de Educación, Universidad Católica de Temuco. Santiago: Frasis editores.

Ramírez, Á. (2001). "Problemas teóricos del conocimiento indígena, presupuestos e inquietudes epistemológicas de base". En: Revista Yachaikuna, № 1, marzo, 4-15.

Ruiz Olabuénaga, J. (1996). Metodología de la investigación cualitativa. Bilbao: Universidad de Deusto.

- (2003). Técnicas de triangulación y control de calidad en la investigación socioeducativa. ( $1^{\mathrm{a}} \mathrm{ed}$.). Bilbao: Ediciones Mensajero.

Sagastizabal, M. (2006). Aprender y enseñar en contextos complejos ( $1^{\text {a }}$ ed.) Buenos Aires: Ediciones Noveduc.

Sabourin, P. (1997). "Perspective sur la mémoire sociale de Maurice Halbwachs". Sociologie et sociétés, Vol. XXIX, № 2, automne, 139-161.

Schnapper, D. (1998). La relation à l'autre. Au coeur de la pensée sociologique. París: Edition Gallimard.

Strauss, A.; Corbin, J. (2002). Bases de la investigación cualitativa. Técnicas y procedimientos para desarrollar la teoría fundamentada. Medellín: Ediciones Universidad de Antioquia.

Taylor, S.; Bogdan, R. (1996). Introducción a los métodos cualitativos de investigación. Barcelona: Ediciones Paidós.

Trinidad, A.; Carrero, V.; Soriano, R. (2006). La construcción de la teoría a través del análisis interpretacional. Madrid: Centro de Investigaciones Sociológicas.

UNESCO (2005). La discriminación y el pluralismo cultural en la Escuela. Casos de Brasil, Chile, Colombia, México y Perú. Santiago de Chile: Oficina Regional de Educación para América Latina y el Caribe OREAL/UNESCO.

Valles, M. (1997). Técnicas cualitativas de investigación social. Reflexión metodológica y práctica profesional. Madrid: Ediciones Síntesis Sociológica. 
Van Dijk, T. (1997). Racismo y análisis crítico de los medios. Londres: John Wiley \& Sons Ltd.

Van Haecht, A. (1999). La escuela va a examen. Preguntas a la sociología de la educación. Buenos Aires: Miño y Dávila Editores.

Villoro, L. (1988). Sobre el concepto de racionalidad. Instituto de Investigaciones Humanísticas. Serie Filosófica, Lima: Editor Juan Camacho.

Wallerstein, I. (2005). Las incertidumbres del saber. Barcelona: Editorial Gedisa, S.A.

Wodak, R. (2003). "De qué trata el análisis crítico del discurso (ACD). Resumen de su historia, sus conceptos fundamentales y sus desarrollos". En: Wodak, Ruth; Meyer, Michel (compiladores) (2003). Métodos de análisis crítico del discurso (pp. 17-34). Barcelona: Editorial Gedisa.

Yáñez, C. (1988). "Estado del arte de la educación indígena en el área andina”. La Educación. Revista Interamericana de Desarrollo Educativo, Año XXXI, No 102, tercer trimestre.

Zambrano, A. (2008). Didáctica, pedagogía y saber. Bogotá: Cooperativa Editorial Magisterio. 